\title{
Experimental Tracheal Replacement: Angiogenesis and Null Apoptosis Promote Stenosis
}

\author{
J. Alfredo Santibáñez-Salgado, M.D. ${ }^{1,2}$, Avelina Sotres-Vega, Ph.D. ', Miguel O. Gaxiola-Gaxiola, M.D. ${ }^{3}$, \\ Jaime Villalba-Caloca, M.D. ', Karen Bobadilla Lozoya, M.D. ${ }^{2,4}$, Joaquín A. Zúñiga-Ramos, Ph.D. ${ }^{2,5}$ \\ 'Lung Transplantation Research Unit, Instituto Nacional de Enfermedades Respiratorias "Ismael Cosio Villegas"; ${ }^{2}$ Tecnológico de Monterrey; ${ }^{3 P a t h o l o g y}$ and \\ Morphology Department, Instituto Nacional de Enfermedades Respiratorias "Ismael Cosio Villegas"; ${ }^{\prime I m m u n o l o g y ~ a n d ~ A m b i e n t a l ~ M e d i c i n e, ~ I n s t i t u t o ~ N a c i o n a l ~ d e ~}$ \\ Enfermedades Respiratorias "Ismael Cosio Villegas"; 5aboratory of Immunobiology and Genetics, Ciudad de México, México
}

\section{ARTICLE INFO}

Received December 2, 2020

Revised March 29, 2021

Accepted April 23, 2021

Corresponding author

J. Alfredo Santibáñez-Salgado

Tel 52-5522704344

Fax 52-5554871700

E-mail alf37@tec.mx

ORCID

https://orcid.org/0000-0002-7632-9158
Background: Tracheal replacement is a challenge for thoracic surgeons due to stenosis in the trachea-prosthesis anastomosis. We propose that stenosis occurs due to fibrosis as a result of an abnormal healing process, characterized by an increased expression of wound healing growth factors (vascular endothelial growth factor [VEGF], survivin, and CD31), which promote angiogenesis and decrease apoptosis. We analyzed the immunoreactivity of VEGF, survivin, CD31, and caspase-3 in the development of fibrotic stenosis in prosthetic tracheal replacement.

Methods: Fourteen dogs were operated on: group I $(n=7)$ received a 6-ring cervical tracheal segment autograft, while in group $\|(n=7)$, a 6 -ring segment of the cervical trachea was resected and tracheal continuity was restored with a Dacron prosthesis. The follow-up was 3 months. Immunoreactivity studies for VEGF, survivin, CD31, and caspase-3 were performed. A statistical analysis was done using the Wilcoxon signed rank test.

Results: Four animals in group I were euthanized on the 10th postoperative day due to autograft necrosis. Three animals completed the study without anastomotic stenosis. Moderate expression of VEGF ( $p=0.038)$, survivin ( $p=0.038)$, and CD31 ( $p=0.038$ ) was found. All group II animals developed stenosis in the trachea-prosthesis anastomotic sites. Microscopy showed abundant collagen and neovascularization vessels. Statistically significant immunoreactive expression of VEGF ( $p=0.015)$, survivin ( $p=0.017)$, and CD31 ( $p=0.011)$ was observed. No expression of caspase-3 was found.

Conclusion: We found a strong correlation between fibrosis in trachea-prosthesis anastomoses and excessive angiogenesis, moderate to intense VEGF, CD31, and survivin expression, and null apoptotic activity. These factors led to uncontrolled collagen production.

Keywords: Trachea, Prosthesis, Angiogenesis, Apoptosis, Fibrosis

\section{Introduction}

Tracheal replacement remains an unsolved challenge in thoracic surgery because tracheal transplantation often fails due to graft necrosis, rejection, or infection [1-5]. A wide variety of prostheses have been studied with limited success, but extensive research in tracheal bioengineering, aiming to produce tracheal replacements based on complex technologies, usually requires a prolonged time to carry out the transplantation. As a result, replacements are limited to short tracheal segments [3]. Despite the limited suc- cess of prostheses, from a surgical and technological point of view, we consider that a prosthesis would be the ideal and easiest way to replace a long segment or the entire trachea, because it would avoid ischemic complications. Nevertheless, at the present time, no predictable and dependable replacement has been found, because prostheses usually present complications such as stenosis (anastomotic and/or luminal), infection, migration, and dehiscence [1-5]. In fact, fibrosis at the trachea-prosthesis anastomotic site is a constant finding. Despite 100 years of research on prosthetic tracheal materials $[1,2,5]$, no studies have addressed 
this complication in depth. In this regard, it has been postulated that the failure of epithelium migration into the prosthesis leads to scar formation, but research has failed to prove this possibility $[1,2,5-7]$. We hypothesized that fibrosis at the trachea-prosthesis anastomotic site results from an abnormal and uncontrolled healing process characterized by active angiogenesis, proliferation of granulation tissue, collagen formation, and the interaction of many healing growth factors [8]. Angiogenesis is a key element of the proliferative phase of healing [8]. Neovascularization is important for healing, supplying oxygen and nutrients, and inducing tissue growth factors to promote the development of different cell lines, resulting in tissue repair. Under certain physiological and pathological situations, angiogenesis is mainly present when there is an increase in tissue mass, a reduction of oxygen levels, or both. Angiogenesis is controlled to a large degree by vascular endothelial growth factor (VEGF) $[8,9]$ and PECAM-1/CD31 (CD31) [10-14]. In addition, survivin inhibits caspase activation and thereby leads to a negative regulation of apoptosis [13]. Survivin is highly expressed in most human tumors and fetal tissue, but is absent in terminally differentiated cells $[15,16]$. The endothelium is one of the most critical sites for the control of apoptosis in vascular injury and vascular remodeling. Inhibition of apoptosis may also be required during vascular remodeling and angiogenesis [1719]. Caspase-3 is a key regulator of apoptosis, and is either partially or fully responsible for the proteolytic cleavage of many key proteins $[18,19]$.

The aim of this work was to assess angiogenesis and the expression of VEGF, CD31, survivin, and caspase-3 associated with the healing process after prosthetic tracheal replacement in an experimental animal model.

\section{Methods}

\section{Ethics statement}

This protocol was reviewed and approved by the Ethics Committee of the Instituto Nacional de Enfermedades Respiratorias "Ismael Cosio Villegas" and was carried out under the technical specifications for the care and use of laboratory animals of the Mexican Official Norm and the Guide for the Care and Use of Laboratory Animals of the National Institutes of Health of the United States of America. The study was approved by the Institutional Review Board of the Comité de Ciencia y Bioética en Investigación (IRB approval no., B30-09).

\section{Prostheses}

A straight 20-mm-diameter Dacron vascular graft (Hemashield Gold; Boston Scientific, Wayne, NJ, USA) was used to restore trachea continuity. For mechanical support of the Dacron graft, external surgical wire rings (Aciflex, surgical stainless-steel suture, Ethicon; Johnson \& Johnson, Langhorne, MO, USA) were fixed with running 6-0 polypropylene (Prolene, Ethicon; Johnson \& Johnson). The ringed graft was sterilized using Sterrad (a low-temperature hydrogen peroxide gas plasma sterilizing process; Johnson \& Johnson Medical Inc., Langhorne, MO, USA) before surgical implantation.

\section{Surgical technique}

Fourteen healthy mongrel dogs chosen regardless of sex or age, weighing from 15 to $20 \mathrm{~kg}$, were operated on. The animals were prepared before surgery with a 12 -hour fast for solids and an 8-hour fast for liquids. Initial anesthesia was induced by intravenous hydrochloric xylazine $(0.1 \mathrm{mg} /$ $\mathrm{kg}$, Rompun; Bayer, Leverkusen, Germany) and propofol (6 $\mathrm{mg} / \mathrm{kg}$, Diprivan; AstraZeneca en México, México, México). Anesthesia was maintained with intravenous propofol ( $2 \mathrm{mg} / \mathrm{kg} / \mathrm{min}$ ), taking care to sustain spontaneous breathing and thus avoid orotracheal intubation. Each anesthetized animal was positioned in the supine position. The neck of the animal was shaved and prepared with povidone-iodine solution. A vertical incision was made in the anterior face of the neck, and the cervical trachea was exposed after separating the strap muscles. In group I $(n=7)$, a 6-ring cervical trachea segment was excised (Fig. 1A), kept in saline solution $(0.9 \%$ sodium chloride; Baxter, Jiutepec, México) for 5 minutes, and re-implanted as an autograft. Proximal and distal end-to-end anastomoses were performed with running 5-0 polypropylene sutures (Prolene, Ethicon; Johnson \& Johnson) (Fig. 1B). In group II $(n=7)$, a 6-ring segment of the cervical trachea was resected. To re-establish airway continuity, a ringed Dacron graft was implanted with proximal and distal end-to-end anastomoses were performed using running 5-0 polypropylene sutures (Fig. 2A).

The animals were allowed to recover spontaneously. All dogs received postoperative intramuscular enrofloxacin (5 $\mathrm{mg} / \mathrm{kg}$, Baytril; Bayer), and metamizole sodium $(28 \mathrm{mg} / \mathrm{kg})$ for one week (Dipiron; Parafarm, México, México). All animals were kept in metabolic cages, and regular observations were made (daily during the first postoperative week and every other day throughout the 3-month study period) 

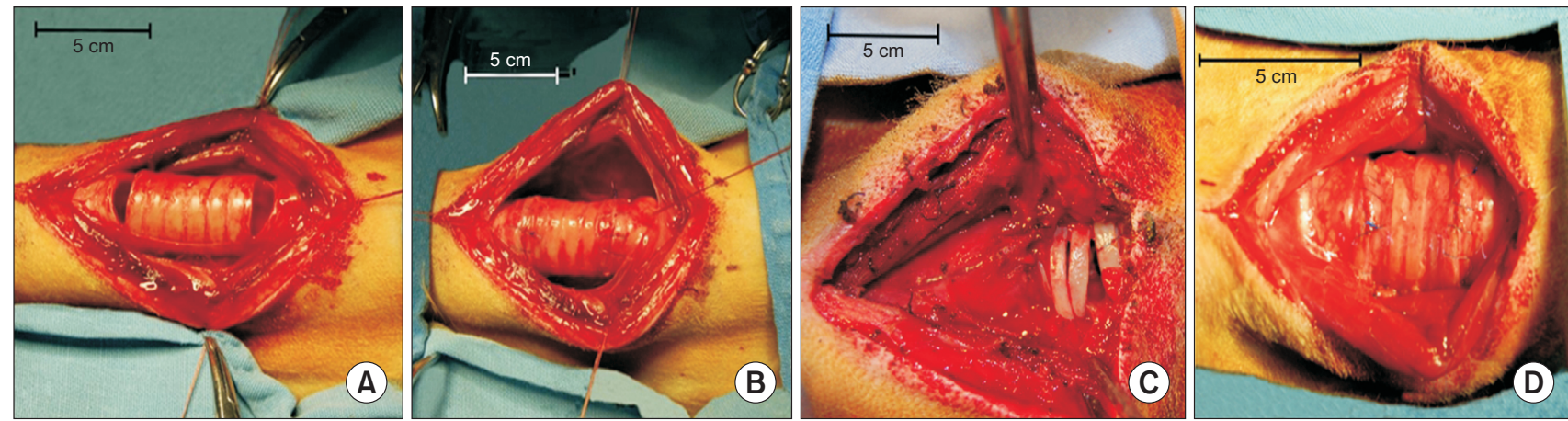

Fig. 1. Native trachea autotransplantation. Clinical course of the autografted trachea. (A) Resection of a 6-ring cervical tracheal segment. (B) Autotransplantation of the native tracheal segment. End-to-end anastomosis with a 5-0 running polypropylene suture. (C) A 6-ring cervical tracheal segment at the moment of euthanasia (10 days after autotransplantation). The tracheal segment shows pale, disrupted tracheal rings due to necrosis. (D) A 6-ring cervical tracheal segment at the moment of euthanasia (3 months after autotransplantation). The autograft is viable. It shows length shortening and mild tortuosity.
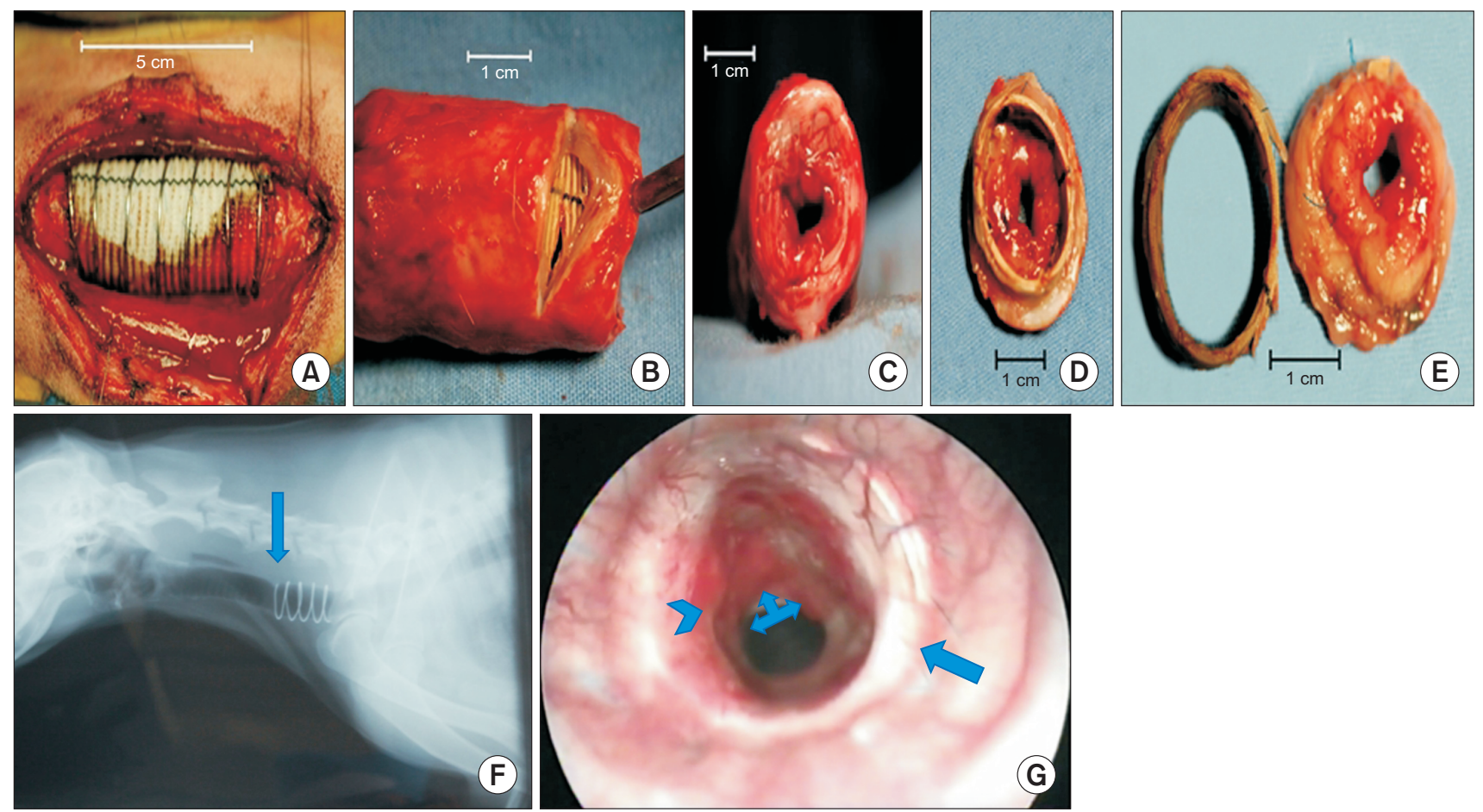

Fig. 2. Prosthetic tracheal replacement with a modified Dacron graft. Clinical course of the Dacron prosthesis. (A) A Dacron prosthesis reinforced with surgical wire used to restore the continuity of the resected 6-ring cervical tracheal segment. (B) The Dacron prosthesis at the moment of euthanasia. The exterior of the prosthesis is completely covered by fibrotic tissue. (C) The prosthesis shows intraluminal fibrous tissue. (D, E) The fibrous tissue was removed from the prosthesis to show the abundant fibrotic growth. (F) Lateral radiograph of the cervical trachea with the Dacron graft prosthesis with the external surgical wire. The arrow shows mild proximal stenosis. Postoperative day 30. (G) Bronchoscopy of the Dacron prosthesis: arrow shows proximal prosthesis trachea anastomosis. The arrow head shows fibrosis in the proximal anastomosis and the triple arrow shows fibrosis in the distal anastomosis. Postoperative day 41.

to detect any post-surgical respiratory signs or clinical complications.

\section{Endoscopic evaluation}

Under intravenous propofol anesthesia as previously de- scribed, the autograft, the prosthesis and native trachea were evaluated immediately after the surgical procedure. Every week, a rigid laparoscope (Surgiview, multi-use $0^{\circ}$, 10-mm diameter; United States Surgical Corp., Norwalk, CT, USA), was used to evaluate changes in the autograft and fibrotic tissue ingrowth at the trachea-prosthesis anas- 
tomotic sites. An image digitalization software program (IMAGE Pro-Plus 4.0; Media Cybernetics, Bethesda, MD, USA) was used to calculate the area. Throughout the study time (in vivo) the stenosis grade was calculated by dividing the internal cut surface area of the stenosed portion of the graft by the native trachea cut surface area on bronchoscopic views. At the end of the study time, the area of the autograft lumen or the fibrotic tissue was traced and compared with the native trachea or prosthesis to obtain the relative final area.

\section{Macroscopic evaluation}

At the time of euthanasia, the surgical area was carefully examined for fibrosis formation around the prosthesis or healing complications (necrosis, dehiscence, fistula formation, and infection as well as graft shrinkage, deformation, or loss). The native trachea with the prosthetic graft was removed. The anastomotic sites were cut and examined carefully on both sides (native trachea and prosthesis) for the development of fibrous tissue or calcifications and to detect respiratory epithelium on the graft.

\section{Microscopic evaluation}

The tissue samples collected from the operated area were fixed in $10 \%$ buffered formalin for 24 hours. They were dehydrated in ascending grades of ethanol, cleared in xylol, embedded and blocked in paraffin wax, and then cut into 4- $\mu \mathrm{m}$-thick sections using a rotatory microtome. The sections were mounted on glass slides and stained with hematoxylin and eosin for general histology, Masson trichrome for the demonstration of collagenous tissue, and Sirius red to identify the type of collagen. Immunoreactivity analyses were used to detect VEGF, CD31, survivin, and caspase-3 immunoreactivity.

The scoring method for angiogenesis activity was to count the newly formed vessels following a modified version of the technique described by Sinicrope [20], where the number of neoformation vessels was determined in at least 5 areas of the slide at 10-fold magnification and assigned one of the following 5 categories: $0-3$, weak; $4-7$, moderate; and $>8$, intense.

\section{Immunohistochemical staining for VEGF, CD31, survivin, caspase-3, and the immunoreactivity scoring method}

Specimens obtained after euthanasia were routinely fixed in $10 \%$ neutral formalin and embedded in paraffin. Serial 4- $\mu \mathrm{m}$-thick sections were cut. In brief, the sections were dewaxed with xylene, rehydrated through graded ethanol, and soaked in Dulbecco's modified phosphate-buffered saline with $\mathrm{Ca}^{2+}$ and $\mathrm{Mg}^{2+}$ (DPBS). The immunoreactivity of VEGF, CD31, survivin, and caspase-3 proteins was evaluated using the standard avidin-biotin-peroxidase complex technique. An antigen retrieval technique (boiling slides at $80^{\circ} \mathrm{C}$ for 15 minutes in a $0.01 \mathrm{M}$ citrate buffer at a $\mathrm{pH}$ of 6.0) was applied and sections were incubated with normal goat serum to block non-specific binding, which was also minimized with several washes with DPBS after each step. The sections were next incubated overnight with the VEGF-A antibody (goat anti-canine VEGF polyclonal antibodies; R\&D Systems, Minneapolis, MN, USA), CD31 antibody (goat anti-canine CD31 polyclonal antibodies; R\&D Systems), survivin antibody (goat anti-canine survivin polyclonal antibody; R\&D Systems), or caspase-3 polyclonal antibody (caspase 3 [CPP32] Ab-4, rabbit polyclonal antibody, Neo Markers; LabVision Co., Fremont, CA, USA) at the indicated concentrations in DPBS. Immune complexes were revealed using biotin-conjugated anti-dog immunoglobulin $\mathrm{G}$ conjugated to streptavidin-peroxidase (Zymed, San Francisco, CA, USA), followed by the chromogen reaction $(0.06 \% 3,3$ '-diaminobenzidine [DAB] tetrahydrochloride, $0.01 \%$ hydrogen peroxide in $\mathrm{DAB})$. Sections were counterstained with hematoxylin and mounted. The slides were examined microscopically using an established scoring system [16,20-22]. A known VEGF, CD31, and survivin-positive slide served as a positive control. Dog tissues that were negative for VEGF, CD31, and survivin were considered as an internal negative control. The intensity of VEGF, CD31, and survivin immunostaining was scored as follows: null, 0; weak, 1+; moderate, 2+; and intense, 3+ $[16,20-22]$.

\section{Detection of apoptosis}

Cell apoptosis was detected using an in-situ apoptosis detection method (terminal deoxynucleotidyl transferase dUTP nick end labeling). The sections were fixed and embedded conventionally. The manipulation was conducted according to the manufacturers' instructions (ApopTag Plus Peroxidase in Situ Apoptosis Detection Kit; Chemicon International Inc., Temecula, CA, USA). The number of apoptotic cells was obtained from the formula for the apoptosis index (AI): $\mathrm{AI}=($ apoptotic cells/total cells $) \times 100 \%$. To detect positive apoptotic cells, candidate positive slides were compared with negative control slides that were treat- 
ed without primary antibody from the ApopTag kit. The slides were analyzed in 5 areas at $\times 10$ magnification. Two independent investigators judged and scored all visualized immunohistochemically stained slides.

\section{Statistical analysis}

Statistical analysis was performed using SPSS ver. 6.1 (SPSS Inc., Chicago, IL, USA). The Wilcoxon signed rank test was used to compare clinical, macroscopic, microscopic, and immunohistochemical findings. All p-values $<0.05$ were considered to indicate statistical significance.

\section{Results}

All animals survived the surgical procedure. Four animals in group I were euthanized on the 10th postoperative day due to partial autograft necrosis. The remaining 3 animals completed the study. All group II animals were followed until the end of the study and were euthanized with an intravenous pentobarbital overdose and $1 \mathrm{mg}$ of $\mathrm{KCl}$.

Four dogs from group I presented mild dyspnea, stridor, and halitosis on postoperative day 7. Dyspnea, stridor, and halitosis became worse on postoperative day 10 , and bronchoscopy confirmed that these animals had developed severe partial autograft necrosis. Thus, for ethical reasons and to avoid suffering, these 4 dogs were euthanized. A macroscopic examination exhibited necrotic autografts with trachea ring disruption, mainly on the middle third of the autografts (Fig. 1C). A microscopic examination revealed necrotic changes in the cartilage and mucosa in the middle third.

The 3 dogs in group I that completed the study had a good clinical course without complications. Endoscopically, the autografts showed a few scattered areas of pale trachea mucosa 7 days after the surgical procedure. After 2 weeks, the autograft mucosa was completely restored.
These 3 animals did not develop fibrosis or stenosis at the anastomotic areas. After euthanasia, the macroscopic examination showed decreases of $2 \mathrm{~mm}, 2 \mathrm{~mm}$, and $3 \mathrm{~mm}$ in the autograft diameter and shortening of $0.9 \mathrm{~cm}, 0.8 \mathrm{~cm}$, and $1.2 \mathrm{~cm}$ in the autograft length, respectively, as well as areas of mild tortuosity (Fig. 1D).

Microscopically, the animals from group I that completed the study without clinical complications exhibited scattered neovascularization vessels. The immunohistochemical analysis found positive expression of VEGF, CD-31 and survivin, negative caspase- 3 expression, and an AI of 0 (Table 1).

Group II: None of the dogs showed dehiscence, fistula formation, infection, graft shrinkage, deformation, or loss. All animals presented with mild dyspnea and stridor when the trachea-prosthesis anastomotic stenosis became $\geq 50 \%$; from the second postoperative week, they presented halitosis.

In the endoscopic evaluation, the graft kept its tubular structure in all the animals. After euthanasia, the macroscopic examination, all the animals showed good prosthesis integration with the native trachea and surrounding cervical structures, without necrosis, fistulae, or infection. The external prosthetic surface was covered with fibrotic tissue (Fig. 2B), without erosion or invasion into neighboring structures (the cervical esophagus, neck vessels, and neck muscles). All the dogs developed fibrotic tissue ingrowth that was confined to the anastomotic sites, which narrowed the trachea-prosthesis anastomotic lumen (Fig. $2 \mathrm{C}-\mathrm{G})$.

Upon microscopic evaluation, there was no evidence of tracheal epithelial growth. The fibrous tissue ingrowth on the trachea-graft anastomosis showed abundant, well-organized collagenous fibers, abundant neovascularization vessels ( $>8 / \times 10$ power field) (Fig. $3 \mathrm{~A}$ ), positive VEGF, CD31, and survivin immunoreactivity, negative caspase-3 expression and an AI of 0 (Fig. 3B-E, Table 2).

Table 1. Control group: trachea autotransplantation

\begin{tabular}{clllccccc}
\hline Dog & VEGF & CD-31 & Survivin & Caspase-3 & Al (\%) & Survived & Stenosis & Fibrosis \\
\hline 1 & + & - & - & - & 0 & No & NA & No \\
2 & ++ & ++ & +++ & - & 0 & Yes & $0 \%$ & No \\
3 & - & - & - & - & 0 & No & NA & No \\
4 & - & - & + & - & 0 & No & NA & No \\
5 & ++ & ++ & +++ & - & 0 & Yes & NA & No \\
6 & + & - & + & - & 0 & No & No \\
7 & ++ & ++ & +++ & - & 0 & Yes & $0 \%$ & \\
\hline
\end{tabular}

$\mathrm{Al}$, apoptosis index; VEGF, vascular endothelial growth factor; -, no immunoreactivity; +, low immunoreactivity; ++, moderate immunoreactivity; +++ , high immunoreactivity; NA, nonassessable. 

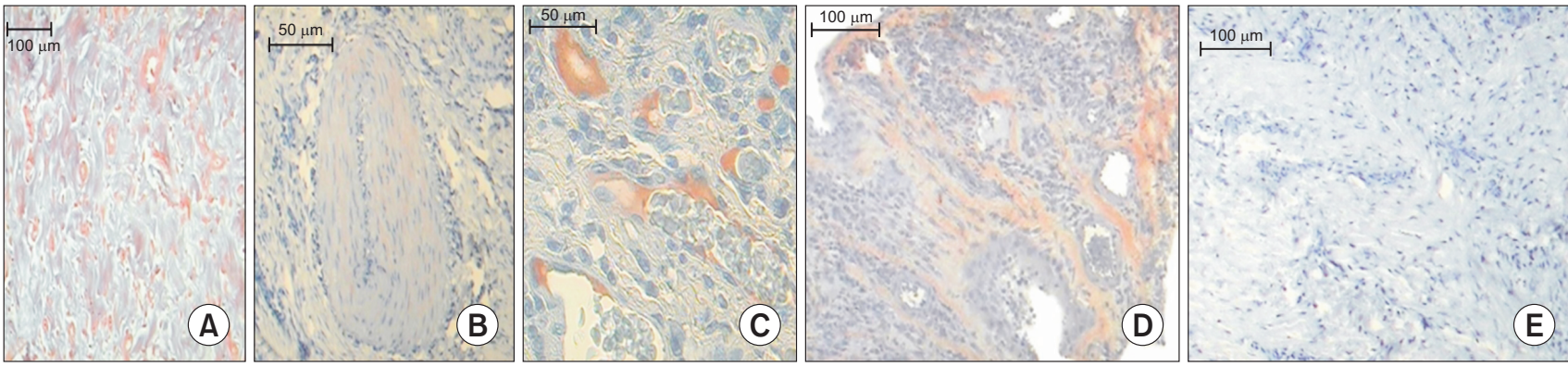

Fig. 3. Immunoreactivity of VEGF, CD31, survivin, and caspase-3 in the fibrotic tissue of the trachea-prosthesis anastomosis sites. (A) A glass slide view of the fibrotic tissue, with abundant collagen fibers and abundant newly formed vessels (in red) $>10 / \times 10$ power field. Hematoxylin and eosin staining. (B) A newly formed vessel positive for vascular endothelial growth factor (red staining in the vessel wall). (C) CD31 is positive in 7 out of 8 newly formed vessels (red staining of the newly formed vessel). (D) Abundant survivin staining (in red) in the collagen fibers. (E) A terminal deoxynucleotidyl transferase dUTP nick end labeling assay was negative for caspase-3 in the fibrotic tissue.

Table 2. Tracheal replacement with modified a Dacron prosthesis: immunoreactivity of VEGF, CD31, survivin, and caspase-3

\begin{tabular}{clllccccc}
\hline Dog & VEGF & CD-31 & Survivin & Caspase-3 & Al (\%) & Survived & Stenosis & Fibrosis \\
\hline 1 & +++ & + & + & - & 0 & Yes & $>50 \%$ & Yes \\
2 & +++ & + & ++ & - & 0 & Yes & $>50 \%$ & Yes \\
3 & + & + & +++ & - & 0 & Yes & $>50 \%$ & Yes \\
4 & +++ & + & ++ & - & 0 & Yes & $>50 \%$ & Yes \\
5 & +++ & + & + & - & 0 & Yes & $>50 \%$ & Yes \\
6 & + & + & +++ & - & 0 & Yes & $>50 \%$ & Yes \\
7 & + & +++ & +++ & - & 0 & Yes & $>50 \%$ & Yes \\
\hline
\end{tabular}

VEGF, vascular endothelial growth factor; Al, apoptosis index; -, no immunoreactivity; +, low immunoreactivity; ++, moderate immunoreactivity; +++ , high immunoreactivity.
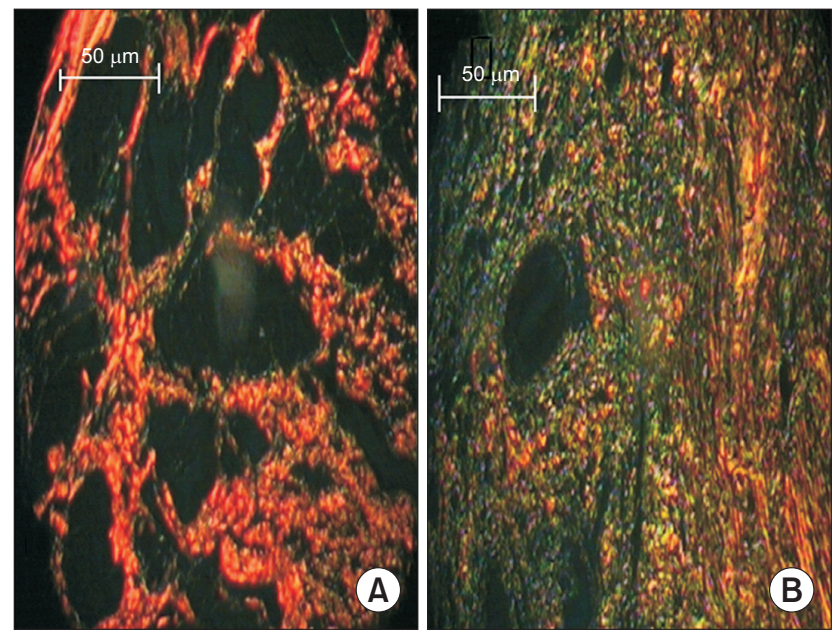

Fig. 4. A glass slide view of fibrotic tissue. (A) Type I collagen fibers (orange areas). (B) Type III collagen fibers (green areas). Sirius red staining with polarization microscopy.

Sirius red staining with polarization microscopy showed that the fibrotic tissue was composed of type I and type III collagen fibers (Fig. 4A, B).

In the animals that received the autograft (group I), VEGF ( $\mathrm{p}=0.038)$ and survivin $(\mathrm{p}=0.038)$ were significantly overexpressed, whereas caspase- 3 was not detected in the tracheal tissue. Similar findings were observed in the animals treated with the Dacron prosthesis (group II). In general, the expression of VEGF was lower than that of survivin $(\mathrm{p}=0.096)$ in group I, but higher than that of survivin $(\mathrm{p}=0.860)$ in group II. We observed that survivin immunoreactivity was significantly higher than $\mathrm{CD} 31$ immunoreactivity in tissues from both groups of animals. In group I, VEGF, survivin, and CD31 were expressed in the 3 dogs that completed the study, whereas VEGF $(\mathrm{p}=0.046)$ and survivin ( $\mathrm{p}=0.038)$ were detected in all dogs from group II that developed fibrosis and stenosis. In addition, caspase-3 was notably absent in all group II dogs $(\mathrm{p}=0.008)$ with stenosis due to fibrosis.

\section{Discussion}

The continued proliferation of scar tissue, leading to obstruction and stenosis, is a common finding in prosthetic tracheal replacement. It has therefore been postulated that the failure of epithelial migration into the prosthesis perpetuates scar formation, but research has failed to demonstrate this possibility [1-7]. We hypothesized that the prolif- 
eration of scar tissue results from a chronic and uncontrolled healing process, with a marked imbalance between angiogenesis and apoptosis. To date, to our knowledge, no study has explored the role of VEGF, survivin, CD31, and apoptosis in the perpetuation of scar formation in prosthetic tracheal replacement.

In this study, we chose Dacron because it is commonly used in clinical practice and possesses good tissue compatibility, and it is known that Dacron prostheses (as well as other prosthetic materials used in tracheal replacement) produce fibrotic stenosis. Therefore, Dacron prostheses were appropriate for investigating the role of these 3 angiogenic factors in fibrosis formation.

We found increased angiogenesis and absent apoptotic activity in the fibrotic scar tissue of trachea-prosthesis anastomotic sites. The 3 proangiogenic factors involved in wound healing (VEGF, CD31, and survivin) studied in this investigation were present in the healing fibrotic tissue. Our findings can be explained as follows: The proliferative phase of healing is characterized by angiogenesis, collagen deposition, granulation tissue formation, epithelialization, and wound contraction [8]. The angiogenesis process occurs concurrently with fibroblast proliferation and migration of endothelial cells to the wound. These cells need oxygen and nutrients, thus making angiogenesis imperative. Tissue in which angiogenesis has occurred appears erythematous due to the presence of capillaries. Eventually, blood vessels and other types of cells that are no longer needed are removed by apoptosis [23]. The fibroblasts produce collagen, and collagen deposition increases wound strength. Type III collagen and fibronectin are produced during the very early stages of wound healing, with deposition peaking during the first 3 weeks, until the later phase of maturation, in which they are replaced by the stronger type I collagen [24].

During angiogenesis, VEGF acts to form new vessels and upregulate survivin, which in turn inhibits caspase-3 [11]. Survivin is undetectable in terminally differentiated adult tissue, but is expressed in human cancers such as stomach, colorectal, lung, breast, pancreatic, and prostate cancers, as well as non-Hodgkin lymphoma. Survivin reduces apoptotic activity, and this reduction is strongly correlated with a poor prognosis in gastric and colorectal cancers. Thus, our findings indicate that during trachea-prosthesis healing, increased angiogenesis resulted from increased VEGF, CD31, and survivin expression and null apoptotic activity, thereby perpetuating the proliferative phase of the healing process and resulting in prolonged angiogenesis and collagen production [24]. The continuous building of fibrotic tissue results in stenosis at the trachea-prosthesis anastomotic sites. As we have mentioned previously, the healing process is a well-orchestrated and complex process, in which many growth factors interact.

The omentum has been successfully used in intrathoracic structures [25]; however, we did not wrap the cervical prosthesis due to the difficulty in reaching the dog's cervical trachea and due to its immunological properties, because it releases angiogenic factors. The omentum has the highest VEGF secretion rate $[25,26]$. It can promote tracheal epithelium growth, but we think that it also leads to excessive granulation tissue, which could explain the presence of stenosis (luminal or anastomotic) in prosthetic tracheal replacement [1-4,7]. It is desirable for the tracheal prosthesis's inner surface to be covered with ciliated respiratory epithelium, but it is not essential, because patients can clear secretions by coughing [1].

Blood supply to the trachea represents a major challenge for trachea transplantation or experimental autotransplantation, because it is usually difficult to restore blood perfusion due to the trachea's fine vascular structures [27]. Experiments with autografts have found that regeneration of the local blood supply is too slow to nourish the complex tissues of the trachea. However, variable results have been reported experimentally with devascularized autografts of different lengths [1-4]. We found that in trachea-trachea (autotransplantation) healing, angiogenesis played a key role in revascularizing the autograft. Revascularization of the autograft was achieved due to the expression of the 3 proangiogenic factors (VEGF, CD31, and survivin), and we can infer that CD31 was the key angiogenic factor, because the autografts that did not express CD31 suffered necrosis. Clinical and experimental non-circumferential autograft tracheal repair (trachea autograft anterior patch plasty) showed freedom from recurrent granulation tissue, in concordance with our results, with the difference that the previous studies applied topical VEGF and we did not $[28,29]$, and we autografted a 6-ring tracheal segment. Dodge-Khatami et al. [30] stated that topical VEGF accelerated autograft revascularization, reduced mucosal fibrosis and inflammation, and preserved normal trachea architecture. Our autografted tracheal segment that became revascularized maintained the tracheal architecture and structure, and was free from stenotic fibrous tissue, probably as a result of not only VEGF expression, but also CD31 and survivin expression, which together exerted a proangiogenic effect and inhibited apoptosis.

We must be very cautious when proposing to use topical VEGF as an angiogenic and healing enhancement factor 
because its excessive use can lead to fibrosis, and has carcinogenic risk; as we found, native VEGF with other native growth factors (CD31 and survivin) could be sufficient to promote angiogenesis, resulting in a normal healing process.

The findings of this study raise many possible research avenues in prosthetic tracheal replacement to try to avoid fibrotic stenosis, which is a catastrophic complication. For example, future studies could seek to identify possible antiangiogenic agents that modulate or stop proangiogenic growth factors, healing modulators, decellularized tracheal scaffolds, or cell culture of tracheal epithelial cells to seed in tracheal prostheses aiming to stop VEGF productionall of which may result in a fibrosis-free anastomosis.

In conclusion, the elevated expression of the proangiogenic factors VEGF, CD31 and survivin, along with the inhibition of apoptosis, and the resultant perpetuation of the healing process resulted in intraluminal obstruction due to excessive fibrotic tissue.

Angiogenesis is the key to obtaining a revascularized autograft. The expression of VEGF, CD31, and survivin promoted angiogenesis on the autograft, maintained its anatomical and functional activity, and avoided fibrosis production.

Further research on healing growth factors, as well as proangiogenic or antiangiogenic factors, must be carried out to obtain a deeper understanding of trachea-prosthesis and trachea-trachea healing processes.

\section{Conflict of interest}

No potential conflict of interest relevant to this article was reported.

\section{ORCID}

J. Alfredo Santibáñez-Salgado: https://orcid.org/0000-0002-7632-9158 Avelina Sotres-Vega: https://orcid.org/0000-0002-1873-4243 Miguel O. Gaxiola-Gaxiola: https://orcid.org/0000-0002-2945-9793 Jaime Villalba-Caloca: https://orcid.org/0000-0001-9499-1930 Karen Bobadilla Lozoya: https://orcid.org/0000-0001-9948-4065 Joaquín A. Zúñiga-Ramos: https://orcid.org/0000-0002-7143-0281

\section{References}

1. Grillo HC. Tracheal replacement: a critical review. Ann Thorac Surg 2002;73:1995-2004

2. Etienne H, Fabre D, Gomez Caro A, et al. Tracheal replacement. Eur Respir J 2018;51:1702211.
3. Delaere P, van Raemdonck D. Tracheal replacement. J Thorac Dis 2016;8(Suppl 2):S186-96.

4. Udelsman B, Mathisen DJ, Ott HC. A reassessment of tracheal substitutes: a systematic review. Ann Cardiothorac Surg 2018;7:175-82.

5. Delaere PR, Vranckx JJ, Den Hondt M; Leuven Tracheal Transplant Group. Tracheal allograft after withdrawal of immunosuppressive therapy. N Engl J Med 2014;370:1568-70.

6. Jorge RG, Armengod AC, Caravajal JM. As originally published in 1990: experimental study of a new porous tracheal prosthesis: updated in 1998. Ann Thorac Surg 1998;65:1830-1.

7. Tsukada H, Osada H. Experimental study of a new tracheal prosthesis: pored Dacron tube. J Thorac Cardiovasc Surg 2004;127:877-84.

8. Nissen NN, Polverini PJ, Koch AE, Volin MV, Gamelli RL, DiPietro LA. Vascular endothelial growth factor mediates angiogenic activity during the proliferative phase of wound healing. Am J Pathol 1998; 152:1445-52.

9. Wilgus TA, Ferreira AM, Oberyszyn TM, Bergdall VK, Dipietro LA. Regulation of scar formation by vascular endothelial growth factor. Lab Invest 2008;88:579-90.

10. DeLisser HM, Christofidou-Solomidou M, Strieter RM, et al. Involvement of endothelial PECAM-1/CD31 in angiogenesis. Am J Pathol 1997;151:671-7.

11. Breen EC. VEGF in biological control. J Cell Biochem 2007;102: 1358-67.

12. Sivakumar B, Harry LE, Paleolog EM. Modulating angiogenesis: more vs less. JAMA 2004;292:972-7.

13. O'Connor DS, Schechner JS, Adida C, et al. Control of apoptosis during angiogenesis by surviving expression in endothelial cells. Am J Pathol 2000;156:393-8.

14. Ferrero E, Ferrero ME, Pardi R, Zocchi MR. The platelet endothelial cell adhesion molecule-1 (PECAM1) contributes to endothelial barrier function. FEBS Lett 1995;374:323-6.

15. Thompson CB. Apoptosis in the pathogenesis and treatment of disease. Science 1995;267:1456-62.

16. Iskandar ZA, Al-Joudi FS. Expression of survivin in fetal and adult normal tissues of rat. Malays J Pathol 2006;28:101-5.

17. Mesri M, Morales-Ruiz M, Ackermann EJ, et al. Suppression of vascular endothelial growth factor-mediated endothelial cell protection by survivin targeting. Am J Pathol 2001;158:1757-65.

18. Cohen GM. Caspases: the executioners of apoptosis. Biochem J 1997;326(Pt 1):1-16.

19. Hotchkiss RS, Strasser A, McDunn JE, Swanson PE. Cell death. N Engl J Med 2009;361:1570-83.

20. Sinicrope FA, Ruan SB, Cleary KR, Stephens LC, Lee JJ, Levin B. bcl-2 and p53 oncoprotein expression during colorectal tumorigenesis. Cancer Res 1995;55:237-41.

21. Lu CD, Altieri DC, Tanigawa N. Expression of a novel antiapoptosis gene, survivin, correlated with tumor cell apoptosis and p53 accumulation in gastric carcinomas. Cancer Res 1998;58:1808-12. 
22. Greenhalgh DG. The role of apoptosis in wound healing. Int J Biochem Cell Biol 1998;30:1019-30.

23. Tanaka K, Iwamoto S, Gon G, Nohara T, Iwamoto M, Tanigawa N. Expression of survivin and its relationship to loss of apoptosis in breast carcinomas. Clin Cancer Res 2000;6:127-34.

24. Stadelmann WK, Digenis AG, Tobin GR. Physiology and healing dynamics of chronic cutaneous wounds. Am J Surg 1998;176(2A Suppl):26S-38S.

25. Mathisen DJ, Grillo HC, Vlahakes GJ, Daggett WM. The omentum in the management of complicated cardiothoracic problems. J Thorac Cardiovasc Surg 1988;95:677-84.

26. Zhang QX, Magovern CJ, Mack CA, Budenbender KT, Ko W, Rosengart TK. Vascular endothelial growth factor is the major angiogenic factor in omentum: mechanism of the omentum-mediated an- giogenesis. J Surg Res 1997;67:147-54.

27. Salassa JR, Pearson BW, Payne WS. Gross and microscopical blood supply of the trachea. Ann Thorac Surg 1977;24:100-7.

28. Backer CL, Mavroudis C, Holinger LD. Repair of congenital tracheal stenosis. Semin Thorac Cardiovasc Surg Pediatr Card Surg Annu 2002;5:173-86.

29. Dodge-Khatami A, Backer CL, Holinger LD, Mavroudis C, Cook $\mathrm{KE}$, Crawford SE. Healing of a free tracheal autograft is enhanced by topical vascular endothelial growth factor in an experimental rabbit model. J Thorac Cardiovasc Surg 2001;122:554-61.

30. Dodge-Khatami A, Niessen HW, Baidoshvili A, et al. Topical vascular endothelial growth factor in rabbit tracheal surgery: comparative effect on healing using various reconstruction materials and intraluminal stents. Eur J Cardiothorac Surg 2003;23:6-14. 